\title{
Информационные, управляющие
}

\author{
и измерительные системы
}

DOI: $10.5862 / J C S T C S .229 .5$

УДК 08-602

О.И. Христодуло, И.Ф. Салимзяноб, Н.Р. ГарееВа

\author{
РАЗРАБОТКА ИНФОРМАЦИОННОЙ СИСТЕМЫ \\ РАЗМЕЩЕНИЯ ОБЪЕКТОВ ТЕХНОГЕННОЙ ОПАСНОСТИ \\ С ИСПОЛЬЗОВАНИЕМ НЕЧЕТКОЙ ЛОГИКИ
}

O.I. Khristodulo, I.F. Salimzynov, N.R. Gareeva

\section{THE DEVELOPMENT OF AN INFORMATION SYSTEM FOR POSITIONING TECHNOGENIC HAZARD OBJECTS USING FUZZY LOGIC}

\begin{abstract}
Описано применение нечеткой логики в информационных системах. Изучены предпосылки использования нечеткой логики, показана математическая модель обработки данных, отображен пример работы информационной системы и сравнительный анализ эффективности ее использования.

СИСТЕМЫ ОБРАБОТКИ ИНФОРМАЦИИ; ИСПОЛЬЗОВАНИЕ НЕЧЕТКОЙ ЛОГИКИ В ГИС; УПРАВЛЕНИЕ ТЕХНОГЕННОЙ БЕЗОПАСНОСТЬЮ; МНОГОКРИТЕРИАЛЬНАЯ МАТЕМАТИЧЕСКАЯ МОДЕЛЬ ДЛЯ ОБРАБОТКИ ДАННЫХ.

This article describes the development of a technogenic hazard management system aimed at landfill positioning. This information system uses fuzzy logic in its operations. The article shows that providing technogenic safety is a fundamental problem in all industrialized countries. One of the main tasks in technogenic safety is landfill positioning, and one of the convenient ways of solving it is using fuzzy logic calculations. The article describes the prerequisites for using fuzzy logic, shows a multicriteria mathematical model for data processing which is normally used in landfill positioning. This model contains a set of linguistic variables describing the input conditions of the analysis and a fuzzy rules base combining these variables. Another model used in the information system is a data processing model developed in ArcGIS Model Builder. The article also presents a logic structure of the system, an example of an information system working and a comparative analysis of the efficiency of its use.

INFORMATION PROCESSING SYSTEMS; USING FUZZY LOGIC IN GIS; TECHNOGENIC SAFETY MANAGEMENT; MULTICRITERIA MATHEMATICAL MODEL FOR DATA PROCESSING.
\end{abstract}

Одна из фундаментальных проблем промышленно развитых стран - обеспечение приемлемого уровня техногенной безопасности. Ошибки, допускаемые на всех уровнях управления техногенной безопасностью, создают угрозу здоровью и жизни населения, являются источником социальной напряженности.

Управление техногенной опасностью в настоящий момент является одним из приоритетных направлений в работе международного сообщества. Растущие объемы промышленного производства провоцируют симметричный рост экологических и техногенных рисков и угроз. Стремление контролировать этот рост привело к наблюдаемому в последние десятилетия экспоненциальному развитию различных про- 
грамм и научных разработок, связанных с техногенной безопасностью.

Объекты техносферы, несущие в себе потенциальную угрозу, разнятся по своему составу, уровню опасности и масштабам распространенности. Помимо действующих промышленных производств, являющихся источниками активной техногенной опасности, необходимо выделить захоронения отходов, которые несут пассивные, нередко отложенные во времени риски. Долговременное воздействие таких захоронений на окружающую среду может спровоцировать серьезные экологические последствия. Будучи промышленно развитым государством, Российская Федерация не является исключением и аккумулирует на своей территории значительное количество промышленных и бытовых отходов (согласно [1], накопленное количество отходов на территории страны превышает 94 млрд т).

В данных условиях важнейшим фактором, влияющим на техногенную опасность, становится правильное размещение объектов захоронения отходов. Однако задача размещения отличается определенной спецификой в связи с неточностью и недостаточностью исходной информации, что отражается на сложности в формулировании достоверных выводов. Разработка методов, позволяющих получать адекватные результаты моделирования о возможном размещении объектов отходов с учетом неопределенности исходной информации и требований к их местоположению, имеет важное практическое значение. Наиболее перспективным при работе в данном направлении видится привлечение экспертных знаний в рамках нечеткой логики.

\section{Использование нечеткой логики при размещении объектов техногенной опасности}

При определении территорий, пригодных для размещения объектов техногенной опасности, учитывается влияние ряда факторов, в соответствии с которыми выносится решение о пригодности территории к применению по целевому назначению. Однако использование строгих правил при классификации территорий снижает гиб- кость и оказывает ограничивающий эффект на процесс принятия управленческих решений, что может привести к росту как социально-экономических, так и техногенных рисков. Более целесообразной является классификация на основе теории нечетких множеств.

Основоположниками теории нечетких множеств являются Л. Заде и Д. Клауа. Авторы расширили понятие множества, допустив, что функция принадлежности элемента множеству может принимать любые значения в интервале от нуля до единицы, а не только нуль или единица [2, 3, 8]. Использование понятия нечеткого множества в задачах классификации обеспечивает большую гибкость при отнесении территории к тому или иному классу, если границы между этими классами имеют переходный характер.

Ядром теории служит понятие нечеткого множества $a$ с функцией принадлежности $\mu a(r)$ и областью значений [0;1]. В данном случае $r \in U-$ множество входных четких значений. Чем больше $\mu a(r)$, тем в большей степени элемент универсального множества соответствует свойствам нечеткого множества. Если $U-$ непрерывное множество, то нечеткое множество $a$ записывается следующим образом:

$$
a=\int_{U}\left(\mu_{a}(r) / r\right) d r
$$

Размещение объектов техногенной опасности осуществляется путем математической формализации нечетких данных, основанных на экспертных методах оценки. Оптимальная позиция объекта определяется характеристикой, называемой лингвистической переменной [4]. Сформулируем постановку задачи для определения степени пригодности территории к размещению на ней объектов техногенной опасности.

Пусть дан набор критериев $k_{i}=\left[k_{1}, \ldots\right.$, $\left.k_{n}\right]$, определяющих оптимальный участок для размещения объектов техногенной опасности. Каждый критерий описывается лингвистической переменной, представленной в виде:

$$
K i=<A i, U i, T i>,
$$


где $A i-$ наименование лингвистической переменной; $U i-$ универсальное множество входных значений; $T i$ - набор возможных значений лингвистической переменной, которые в рамках исследования размещения объектов техногенной опасности представлены как «Абсолютно пригодно», «Пригодно» и др.

Требуется найти выходной параметр, определяющий степень пригодности территории к размещению объекта техногенной опасности, который описывается лингвистической переменной $y$. Необходимо определить влияние каждой из пяти входных лингвистических переменных на выбор участка территории, для чего следует сформировать базу правил нечеткого вывода [9]. Такие правила устанавливаются при помощи зависимостей, представляющих собой логические высказывания вида ЕСЛИ $A$, то $B$ :

$$
P: A \rightarrow B,
$$

где $P$ - условие применимости ядра (обычно представляет собой логическое выражение).

При проведении пространственного анализа на основе нечеткой логики важным аспектом является выбор функции принадлежности и ее параметров для нечетких множеств $[5,10]$. В практической деятельности применяются следующие виды функций принадлежности:

треугольные и трапециевидные (линейно-кусочные);

нелинейные (функция Гаусса, сигмоидальная функция, сплайн);

LR-представление функций принадлежности.

Использование этих функций сокращает численные расчеты и, соответственно, вычислительные ресурсы, необходимые для хранения отдельных значений функции принадлежности.

\section{Многокритериальная математическая модель размещения объектов техногенной опасности на основе нечеткой логики}

Согласно нормативным актам, в которых указаны основные требования к месту расположения полигонов отходов, на вы- бор территории для размешения полигона накладывается ряд ограничений [6]:

расстояние до населенных пунктов и открытых водоемов устанавливается с учетом конкретных местных условий, но не менее $3000 \mathrm{M}$;

расстояние от границ леса и лесопосадок, не предназначенных для использования в рекреационных целях, - не менее 50 м;

глубина залегания грунтовых вод - не менее 2 м от нижнего уровня захороненных отходов;

размещение полигонов не допускается в зонах активного карста.

Отметим, что перечисленные требования являются минимально необходимыми. На выбор территории также влияют долгосрочные перспективы эксплуатации объекта захоронения отходов, такие как изменение состава хранимых отходов, увеличение площади полигона и др.

Для достижения максимальных эксплуатационных возможностей математической модели, а также с целью обеспечения корректности нечеткой базы знаний, к разработке модели были привлечены специалисты отдела экологической безопасности Министерства природопользования и экологии Республики Башкортостан. Группа экспертов, состоящая из сотрудников отдела, работала в соответствии с прямым методом построения [11], согласно которому эксперт непосредственно задает значение функции принадлежности (степень соответствия элемента $r$ ) для каждого значения исследуемого параметра.

Значения функции принадлежности вычислялись по формуле:

$$
\mu_{A}=\frac{n_{A}}{n},
$$

где $n$ - число экспертов, участвовавших в эксперименте; $n_{A}$ - число экспертов, проголосовавших за соответствие элемента $r$ нечеткому множеству $A$.

По полученным в результате экспертного анализа значениям функции фиксируются характерные точки. Следующий этап аппроксимация подходящей типовой функцией по характерным точкам. Нечеткие подмножества, полученные в результате 
этих операций, ассоциируются с определенным термом из формализованного экспертами терм-множества.

В разработанной модели использовались следующие типы функций: S-образные, $\mathrm{Z}$-образные и сигмоидальные функции

Требования к месту расположения полигонов отходов были выражены через следующие лингвистические переменные:

расстояние до грунтовых вод;

расстояние до карстовых пород;

расстояние до населенных пунктов;

расстояние до водных объектов;

расстояние до лесного массива.

Фрагмент модели, соответствующей лингвистической переменной «Глубина залегания грунтовых вод», описан выражениями (5), (6). Лингвистическая переменная обладает тремя значениями, или термами «Недопустимо близко», «Близко» и «Далеко», - каждый из которых описывается соответствующей функцией принадлежности. Таким образом, из рис. 1 видно, что степень принадлежности, например, терму «Близко» снижается при изменении глубины залегания грунтовых вод с 2 до 4 м. Соответственно, в этом же диапазоне возрастает степень принадлежности переменной к терму «Далеко».

Переменная «Расстояние до грунто-
вЫХ вод»:

$$
\begin{aligned}
& A_{1}^{I \mathrm{\Gamma \textrm {B }}} \in T_{1}^{I \mathrm{\Gamma \textrm {B }}}: T_{1}^{I \mathrm{\Gamma \textrm {B }}}= \\
& =\left\{\begin{array}{c}
a_{1,1}-\text { Недопустимо близко, } \\
a_{1,2}-\text { Близко, } \\
a_{1,3}-\text { Далеко }
\end{array}\right\} \text {, } \\
& \mu_{1,1}=\left\{\begin{array}{l}
1, r_{1} \leq 2, \\
0, r_{1}>2
\end{array},\right. \\
& \mu_{1,2}=\left\{\begin{array}{c}
\frac{1}{2}+\frac{1}{2} \cos \left(\frac{r_{1}-2}{2} \pi\right), 2<r_{1} \leq 4, \\
0, r_{1}>4
\end{array}\right. \\
& \mu_{1,3}=\left\{\begin{array}{c}
0, r_{1}<2, \\
\frac{1}{2}+\frac{1}{2} \cos \left(\frac{r_{1}-4}{2} \pi\right), 2 \leq r_{1} \leq 4, \\
1, r_{1}>4
\end{array}\right.
\end{aligned}
$$

Другой критерий, описанный в рамках многокритериальной математической модели, «Расстояние до населенных пунктов» формализован формулами (7), (8). В отличие от предыдущего критерия, отображенного на рис. 1, данный критерий обладает четырьмя лингвистическими термами (рис. 2).

Переменная «Расстояние до населенных пунктов»:

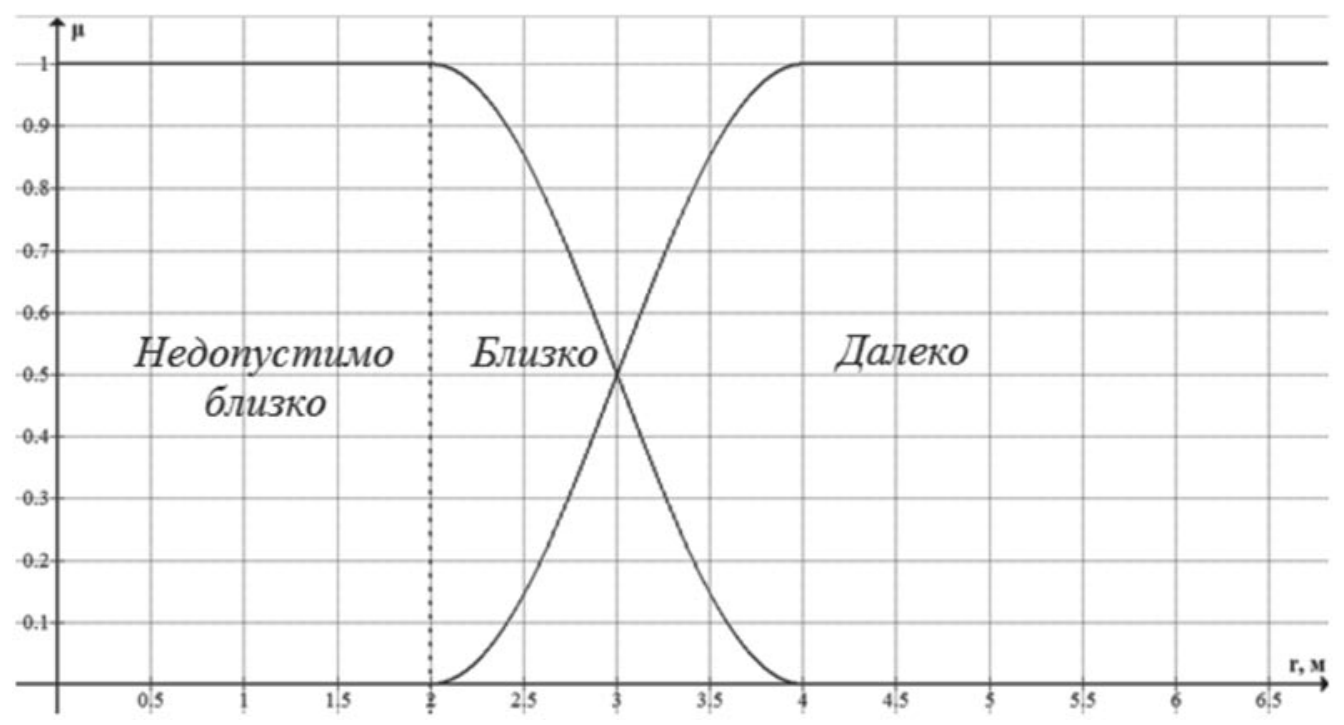

Рис. 1. Функция принадлежности для термов лингвистической переменной «Расстояние до грунтовых вод» 


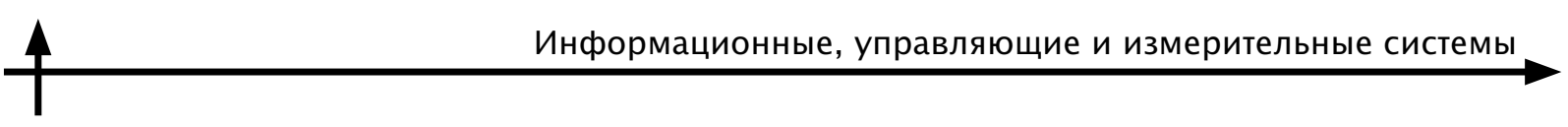

$$
\begin{aligned}
& A_{3}^{\mathrm{HH}} \in T_{3}^{\mathrm{H \textrm {HI }}}: T_{3}^{\mathrm{LH}}= \\
& =\left\{\begin{array}{c}
a_{3,1}-\text { Недопустимо близко, } \\
a_{3,2}-\text { Близко, } \\
a_{3,3}-\text { Недалеко, } \\
a_{3,4}-\text { Далеко }
\end{array}\right\} \text {. } \\
& \mu_{3,1}=\left\{\begin{array}{l}
1, r_{3} \leq 3000 \\
0, r_{3}>3000
\end{array}\right. \\
& \mu_{3,2}=\frac{1}{1+e^{\left(0,012\left(r_{3}-3500\right)\right.}}, \\
& \mu_{3,3}= \begin{cases}\frac{1}{1+e^{\left(-0,012\left(r_{3}-3500\right)\right)}}, & r_{3} \leq 4000, \\
\frac{1}{1+e^{\left(0,012\left(r_{3}-4500\right)\right)}}, & r_{3}>4000\end{cases} \\
& \mu_{3,4}=\frac{1}{1+e^{\left(-0,012\left(r_{3}-4500\right)\right.}} .
\end{aligned}
$$

Полная многокритериальная математическая модель размещения объектов техногенной опасности на основе нечеткой логики приведена в [7]. Необходимо отметить, что разработанная модель позволяет вводить неограниченное число критериев, например, преобладающее направление ветра, уклон поверхности и др.

На основе экспертных знаний была сформирована база правил, определяющая степень пригодности анализируемой территории для размещения на ней объекта техногенной опасности для захоронения отходов (см. табл.).

Для автоматизации обработки данных разработана модель в среде ArcGIS Model Builder. Алгоритм обработки основан на процедуре нечеткого логического вывода и состоит из следующих этапов:

- преобразование векторных поверхностей в растры;

- объединение части растров (необходимо для снижения временных затрат на обработку данных);

- фаззификация (перевод характеристик в нечеткую форму с помощью функций принадлежности);

- агрегирование (определение степени истинности условия по каждому правилу);

- активизация подусловий (объединение полученных на этапе агрегирования степеней истинности);

- дефаззификация (преобразование нечетких данных обратно в четкую форму);

- представление результатов.

На рис. 3 представлена общая структура модели и отдельно вынесен этап представления результатов. На этом этапе формируются поверхности, соответствующие термам выходной лингвистической пере-

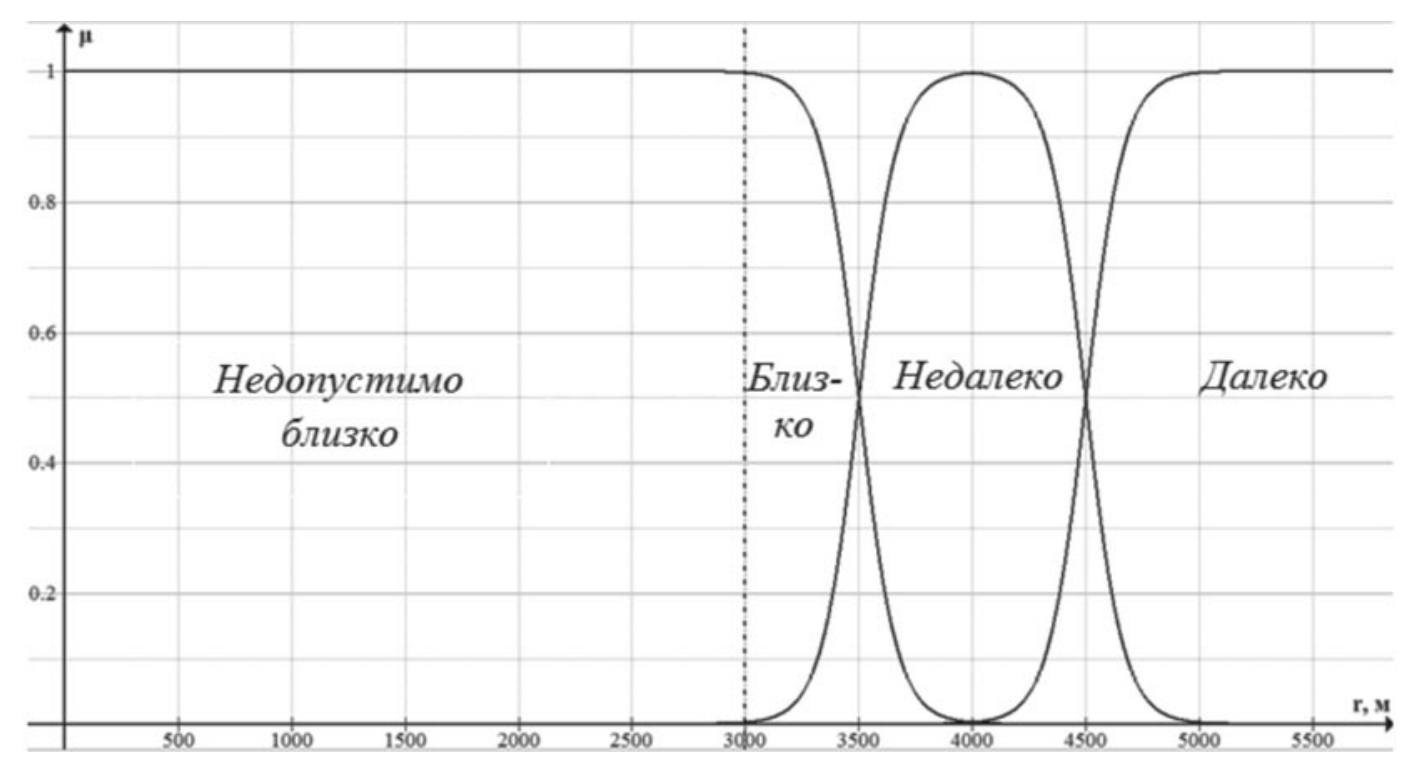

Рис. 2. Функция принадлежности для термов лингвистической переменной «Расстояние до населенных пунктов» 
База правил

\begin{tabular}{|c|c|c|c|c|c|c|}
\hline & ЕСЛИ $r_{1}=A_{1}$ & И $r_{2}=A_{2}$ & И $r_{3}=A_{3}$ & $И r_{4}=A_{4}$ & $И r_{5}=A_{5}$ & TO $y=B_{i}$ \\
\hline 1 & далеко & далеко & далеко & далеко & далеко & \multirow{5}{*}{$\begin{array}{c}\text { Абсолютно } \\
\text { пригодна }\end{array}$} \\
\hline 2 & далеко & далеко & далеко & далеко & близко & \\
\hline 3 & далеко & далеко & далеко & близко & далеко & \\
\hline 4 & далеко & далеко & близко & далеко & далеко & \\
\hline 5 & далеко & далеко & недалеко & далеко & далеко & \\
\hline 6 & далеко & далеко & недалеко & далеко & близко & \multirow{16}{*}{ Пригодна } \\
\hline 7 & далеко & далеко & недалеко & близко & далеко & \\
\hline 8 & далеко & близко & недалеко & далеко & далеко & \\
\hline 9 & близко & далеко & недалеко & далеко & далеко & \\
\hline 10 & далеко & далеко & далеко & близко & близко & \\
\hline 11 & далеко & близко & далеко & далеко & далеко & \\
\hline 12 & далеко & близко & далеко & далеко & близко & \\
\hline 13 & далеко & близко & далеко & близко & далеко & \\
\hline 14 & близко & далеко & далеко & далеко & далеко & \\
\hline 15 & близко & далеко & далеко & далеко & близко & \\
\hline 16 & близко & далеко & далеко & близко & далеко & \\
\hline 17 & близко & близко & далеко & далеко & далеко & \\
\hline 18 & далеко & далеко & близко & далеко & близко & \\
\hline 19 & далеко & далеко & близко & близко & далеко & \\
\hline 20 & далеко & близко & близко & далеко & далеко & \\
\hline 21 & близко & далеко & близко & далеко & далеко & \\
\hline 22 & далеко & близко & далеко & близко & близко & \multirow{16}{*}{$\begin{array}{c}\text { Недостаточно } \\
\text { пригодна }\end{array}$} \\
\hline 23 & близко & далеко & далеко & близко & близко & \\
\hline 24 & близко & близко & далеко & далеко & близко & \\
\hline 25 & близко & близко & далеко & близко & далеко & \\
\hline 26 & далеко & далеко & близко & близко & близко & \\
\hline 27 & далеко & близко & близко & далеко & близко & \\
\hline 28 & далеко & близко & близко & близко & далеко & \\
\hline 29 & близко & далеко & близко & далеко & близко & \\
\hline 30 & близко & далеко & близко & близко & далеко & \\
\hline 31 & близко & близко & близко & далеко & далеко & \\
\hline 32 & далеко & далеко & недалеко & близко & близко & \\
\hline 33 & далеко & близко & недалеко & далеко & близко & \\
\hline 34 & далеко & близко & недалеко & близко & далеко & \\
\hline 35 & близко & далеко & недалеко & далеко & близко & \\
\hline 36 & близко & далеко & недалеко & близко & далеко & \\
\hline 37 & близко & близко & недалеко & далеко & далеко & \\
\hline 38 & близко & близко & далеко & близко & близко & \multirow{3}{*}{ Непригодна } \\
\hline 39 & далеко & близко & близко & близко & близко & \\
\hline 40 & близко & далеко & близко & близко & близко & \\
\hline
\end{tabular}




\begin{tabular}{|c|c|c|c|c|c|c|}
\hline & ЕСЛИ $r_{1}=A$ & И $r_{2}=A_{2}$ & И $r_{3}=A_{3}$ & И $r_{4}=A_{4}$ & И $r_{5}=A_{5}$ & TO $y=B$ \\
\hline 41 & близко & близко & близко & далеко & близко & \multirow{8}{*}{ Непригодна } \\
\hline 42 & близко & близко & близко & близко & далеко & \\
\hline 43 & близко & близко & близко & близко & близко & \\
\hline 44 & близко & близко & недалеко & близко & близко & \\
\hline 45 & далеко & близко & недалеко & близко & близко & \\
\hline 46 & близко & далеко & недалеко & близко & близко & \\
\hline 47 & близко & близко & недалеко & далеко & близко & \\
\hline 48 & близко & близко & недалеко & близко & далеко & \\
\hline
\end{tabular}

менной и характеризующие степень пригодности территории для размещения объектов техногенной опасности.

\section{Информационная система размещения объектов техногенной опасности в условиях неопределенности}

В целях автоматизации процесса определения степени пригодности территории для размешения объектов техногенной опасности разработана информационная система, в работе которой применяется нечеткая логика. Данная информационная система решает следующие задачи:

определение степени пригодности выбранного участка территории для размещения объектов техногенной опасности на основании заданных критериев;

подготовка отчетной документации в виде карты и ее экспорт в пригодный для печати формат; автоматизация процесса определения степени пригодности выбранного участка территории для размещения объектов техногенной опасности путем снижения трудоемкости осушествляемых операций;

снижение требований к квалификации специалистов, осуществляющих анализ.

Структура информационной системы включает в себя базы пространственной и атрибутивной информации, содержащие общегеографическую информацию, необходимую для анализа, данные об объектах техногенной опасности, а также базу нечетких знаний (рис. 4).

В качестве примера работы информационной системы на рис. 5 отображен участок территории, на котором был осуществлен анализ степени пригодности территории для размещения объектов твердых бытовых отходов. В результате автоматизированной обработки исходных данных получены ито-
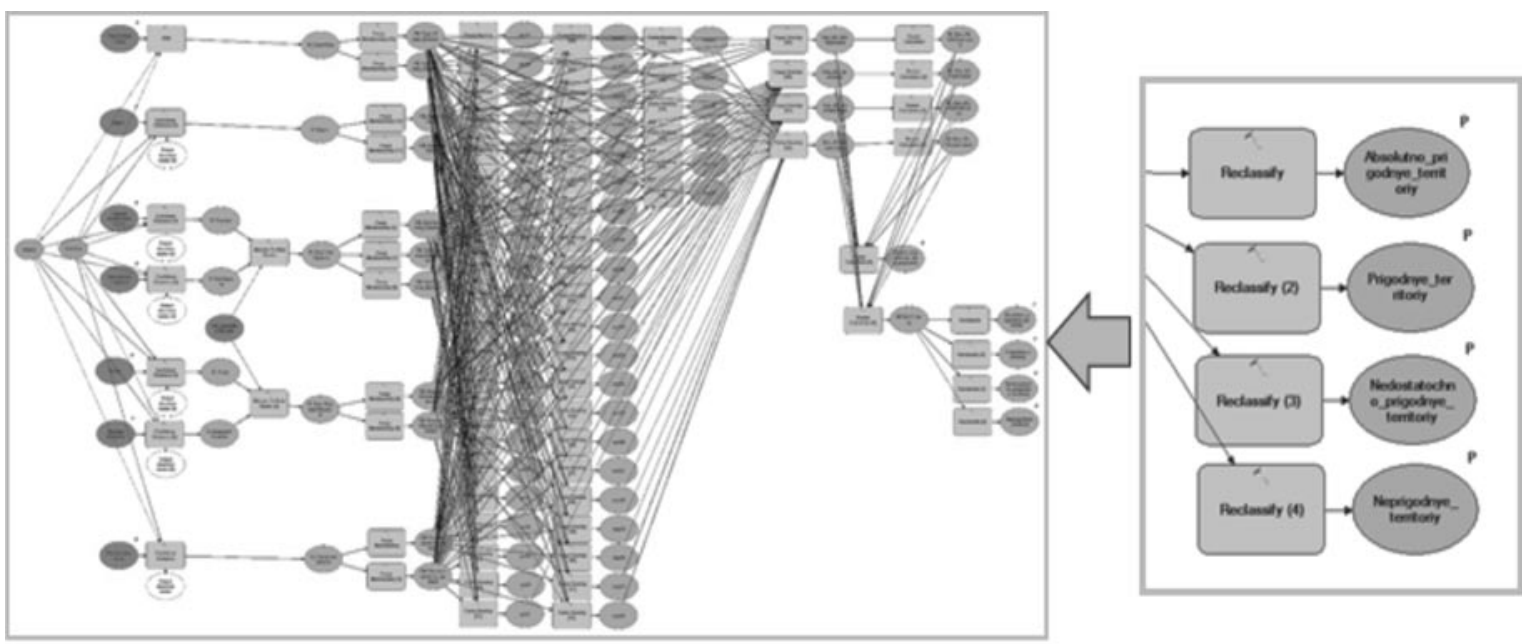

Рис. 3. Модель обработки данных в ARCGIS MODEL BUILDER 


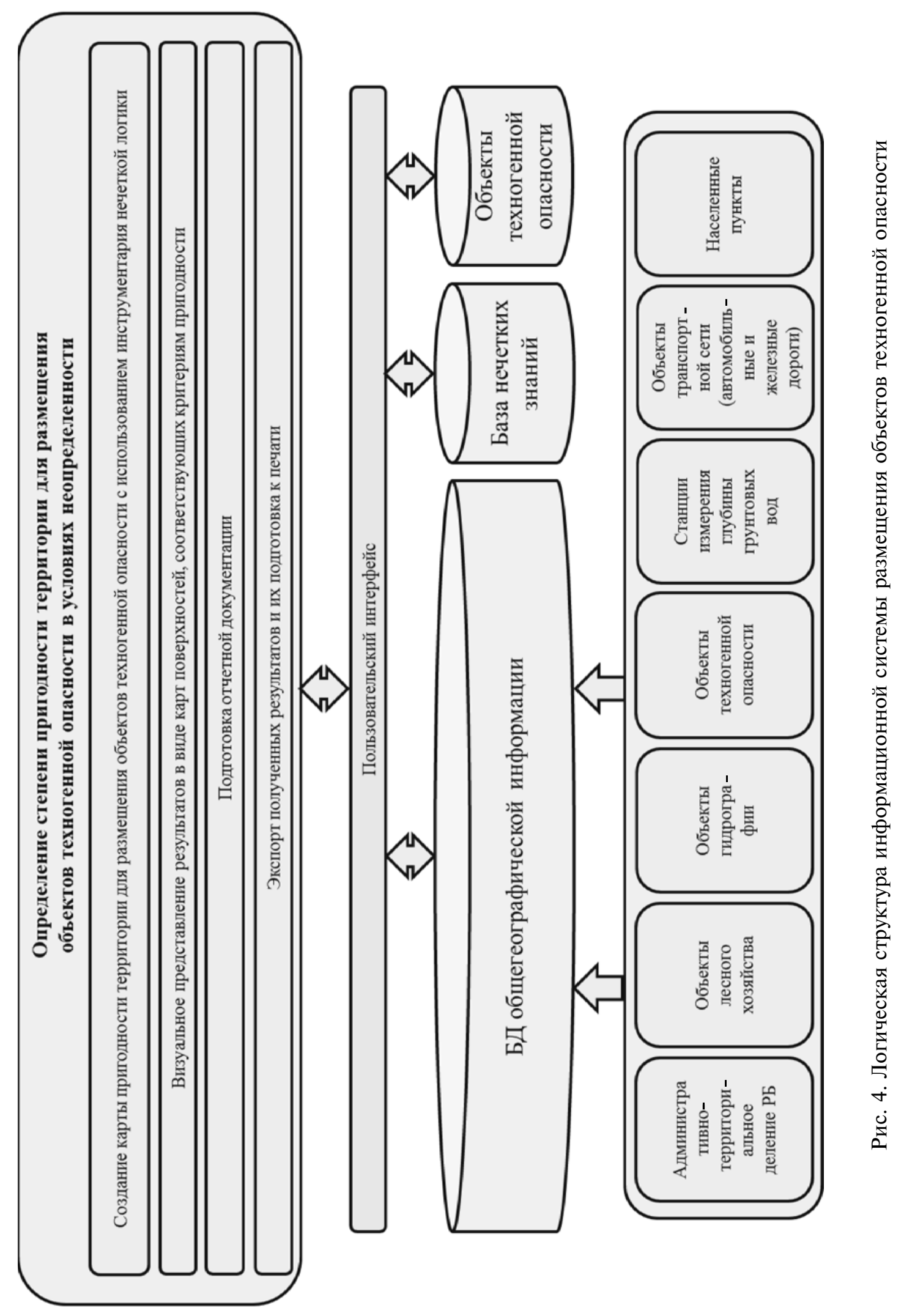




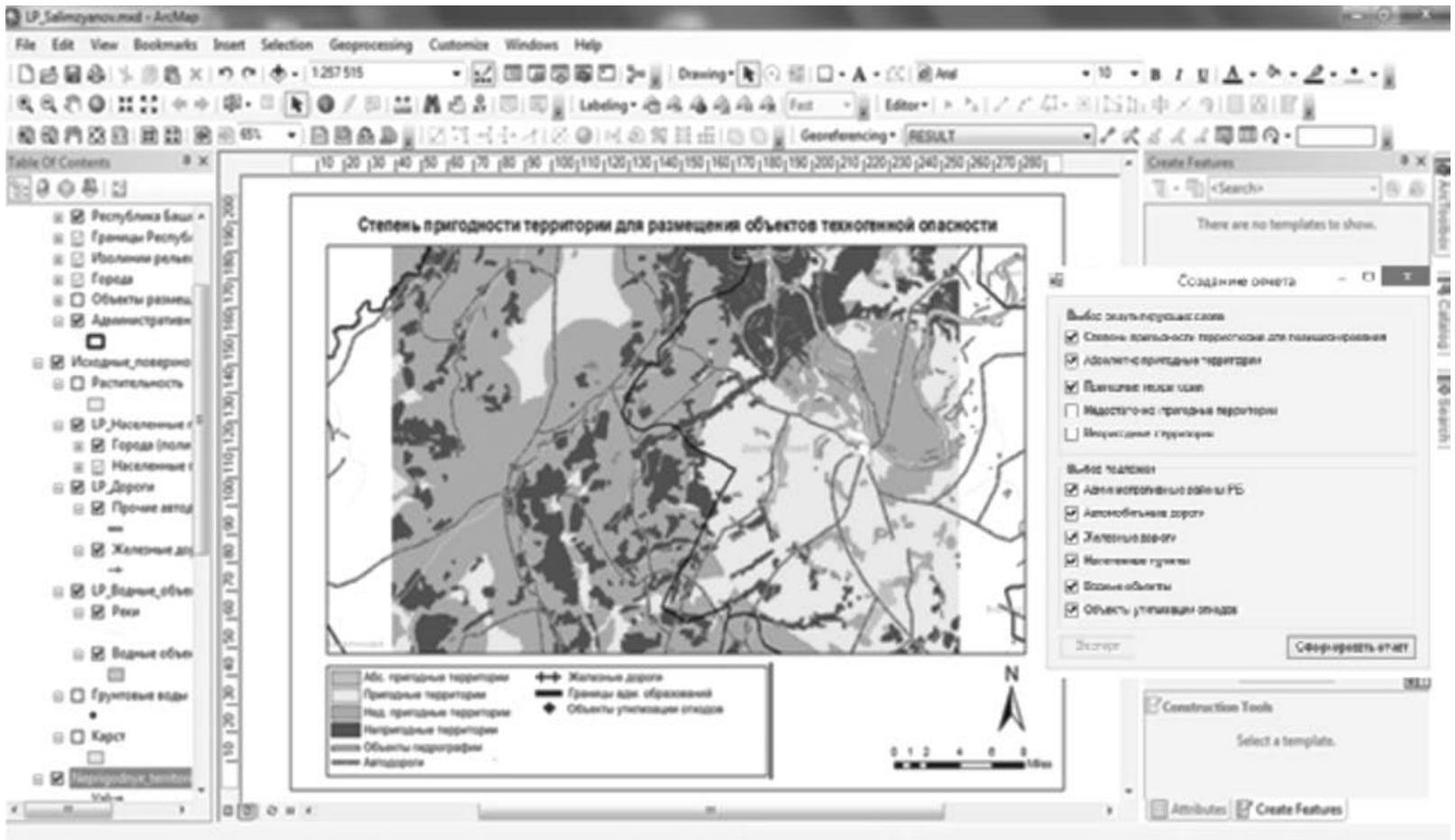

Рис. 5. Пример анализа степени пригодности территории для размещения объектов твердых бытовых отходов

говые растры, отображающие соответствие участков территории соответствующим термам (критериям) выходной лингвистической переменной, при этом формируется карта с легендой и условными обозначениями. Например, светло-зеленые участки территории являются наиболее пригодными для размещения объектов техногенной опасности. Кроме того, интерфейс системы позволяет управлять отображением отдельных результирующих поверхностей (например, если требуется отобразить на карте только непригодные для размещения объектов техногенной опасности территории) и слоев подложки, таких как дороги, водные объекты и др.

На основании данных, полученных от специалистов Минэкологии РБ, - лиц, потенциально заинтересованных в использовании разработанной системы, - и временных затрат на автоматизированную обработку данных был проведен сравнительный анализ эффективности разработанной информационной системы, согласно которому для обработки территории, соответствующей одному муниципальному району Республики Башкортостан, требуется 62,5 мин для автоматизированной обработки данных, против 107,5 мин при ручной обработке.

Достигнута экономия времени порядка $40 \%$ (рис. 6). При экстраполяции на всю территорию Республики Башкортостан экономия времени достигает 47 ч.

Другим преимуществом системы являются более низкие требования к квалификации специалиста, осуществляющего анализ, т. к. экспертные знания, необходимые для обработки, уже формализованы.

Размещение объектов техногенной опасности имеет важное практическое значение, связанное с необходимостью контроля над экологическими рисками и возможными последствиями техногенного характера. Разработанная информационная система объектов техногенной опасности в условиях неопределенности позволяет осуществлять размещение объектов техногенной опасности по заданным критериям, обеспе- 


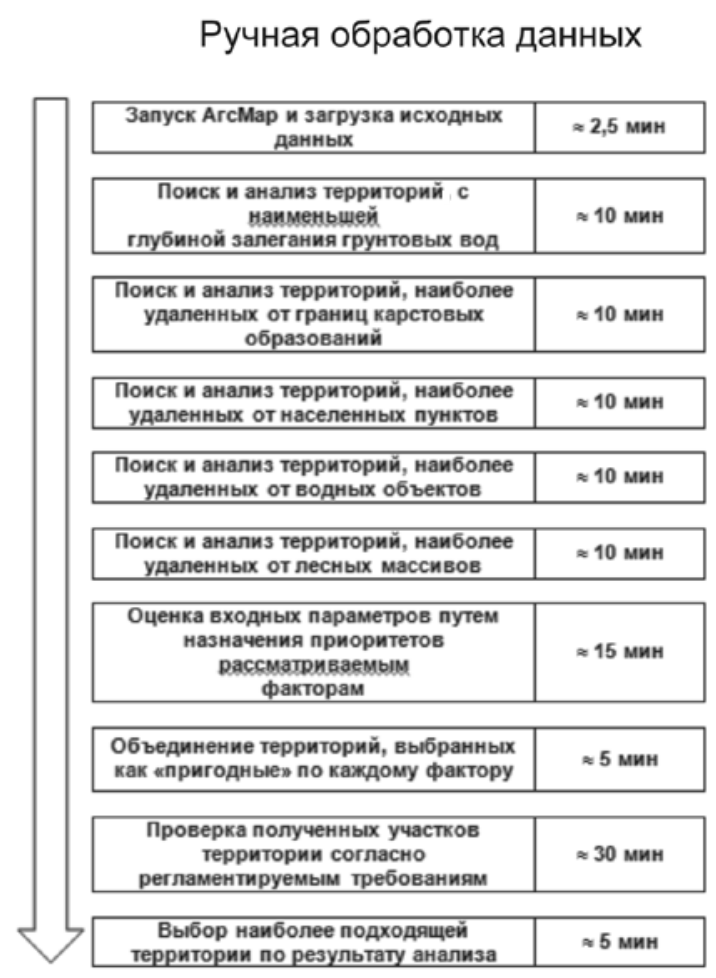

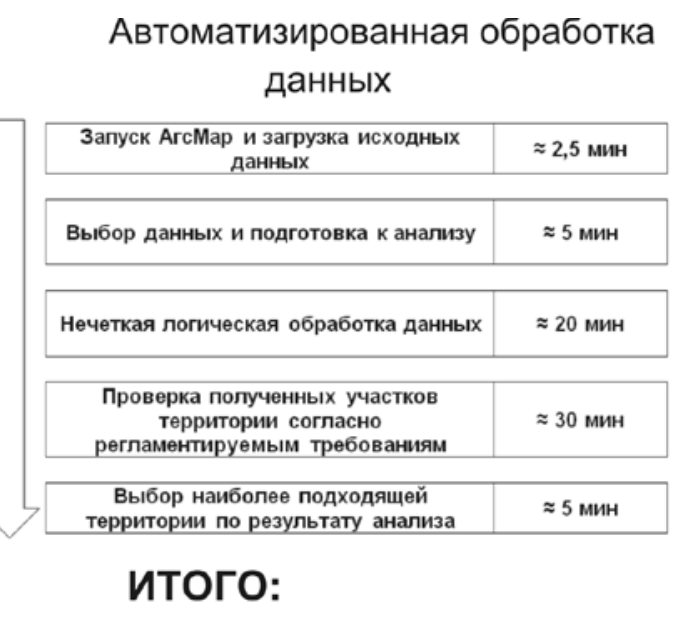

Ручная обработка данных: Автоматизированная обработка данных:
107,5 мин

62,5 мин

Рис. 6. Анализ эффективности информационной системы объектов техногенной опасности в условиях неопределенности

чивая формализацию экспертных знаний и позволяя сократить временные затраты на проводимый анализ.

В настоящий момент функциональность системы расширяется в части возможности динамического изменения критериев анализа и пороговых значений термов функции принадлежности. Внедрение дополнительного функционала позволит использовать информационную систему для анализа территории в любых целях, не только для размещения полигонов отходов, и позволит учитывать специфические особенности территории, которая будет подвергнута анализу. K примеру, для гористой местности возможно добавление такого критерия, как частота и сила оползней по шкале Медведева-Шпонхойера-Карника.
Однако дальнейшее использование системы возможно и без расширения ее функциональности. Существующая модель обработки данных пригодна для анализа всей территории Российской Федерации или других стран, а выигрыш по времени при анализе участков территории таких масштабов с помощью информационной системы по сравнению с ручной обработкой информации возрастает пропорционально площади исследуемой территории.

Результаты исследований, представленные в статье, частично поддержаны грантом № 15-08-01758-A «Методологические и методические основы анализа техногенной безопасности в условиях неопределенности состояния объектов управления».

\section{СПИСОК ЛИТЕРАТУРЫ}

1. Федеральная служба государственной статистики. Образование отходов производства и потребления по видам экономической деятельности [электронный ресурc]/ URL: http://www. gks.ru/free_doc_new_site/oxrana/tabl/oxr_otxod1. xls/ (дата обращения: 19.12.2014).

2. Zadeh L.A. Fuzzy sets // Information and Control. 1965. Vol. 8. No. 3. Pp. 338-353.

3. Klaua D., Gottwald S. An early approach toward graded identity and graded membership in set 
theory // Fuzzy Sets and Systems. Vol. 161, No. 18. Pp. 114-119.

4. Давлетбакова 3.Л. Применение методов нечеткой логики в пространственном анализе объектов размещения отходов // Актуальные проблемы науки и техники. Информационные и инфокоммуникационные технологии, естественные науки: сб. трудов. Уфа: Изд-во УГАТУ, 2012. T. 1. C. $303-306$.

5. Алтунин А.Е., Семухин М.В. Модели и алгоритмы принятия решений в нечетких условиях: Монография. Тюмень: Изд-во Тюменского гос. ун-та, 2000. 352 с.

6. Санитарно-защитные зоны и санитарная классификация предприятий, сооружений и иных объектов // СанПиН 2.2.1/2.1.1.1200-03. Зарег. в Мин-ве юстиции РФ 29.04.2003. № 4459.

7. Христодуло О.И., Салимзянов И.Ф., Гареева Н.P. Многокритериальная математическая модель позиционирования объектов техногенной опасности на основе нечеткой логики //
Геоинформационные технологии в проектировании и создании корпоративных информационных систем: межвуз. науч. сб. Уфа: Изд-во УГАТУ, 2015. С. 55-64.

8. Заде Л. Понятие лингвистической переменной и его применение к принятию приближенных решений. М.: Мир, 1976. 166 с.

9. Штовба С.Д. Введение в теорию нечетких множеств и нечеткую логику. Винница: Континент-Прим, 2003. 198 с.

10. Зак Ю.А. Принятие решений в условиях нечетких и размытых данных: Fuzzyтехнологии. М.: Книжный дом «ЛИБРОКОМ», 2013. 352 c.

11. Павлов С.В., Давлетбакова 3.Л., Абдуллин А.X. Информационная система поддержки принятия решений по управлению отходами на территории Республики Башкортостан на основе базы нечетких знаний // Электротехнические и информационные комплексы и системы. 2013. № 4. T. 9. С. 113-120.

\section{REFERENCES}

1. Federalnaya sluzhba gosudarstvennoy statistiki. Obrazovaniye otkhodov proizvodstva $i$ potrebleniya po vidam ekonomicheskoy deyatelnosti [Federal State Statistics Service. Waste production and consumption by economic activity]. Available: http://www.gks. ru/free_doc_new_site/oxrana/tabl/oxr_otxod1.xls/ (Accessed: 19.12.2014). (rus)

2. Zadeh L.A. Fuzzy sets. Information and Control, 1965, Vol. 8, No. 3, Pp. 338-353.

3. Klaua D., Gottwald S. An early approach toward graded identity and graded membership in set theory. Fuzzy Sets and Systems, Vol. 161, No. 18, Pp. 114-119.

4. Davletbakova Z.L. Primeneniye metodov nechetkoy logiki $\mathrm{v}$ prostranstvennom analize obyektov razmeshcheniya otkhodov [Application of fuzzy logic in the spatial analysis of waste disposal facilities]. Aktualnyye problemy nauki i tekhniki: Informatsionnyye $i$ infokommunikatsionnyye tekhnologii, yestestvennyye nauki [Actual problems of science and technology and information and communication technology Information, Science], Ufa: UGATU Publ., 2012, Vol. 1, Pp. 303-306. (rus)

5. Altunin A.Ye., Semukhin M.V. Modeli $i$ algoritmy prinyatiya resheniy $v$ nechetkikh usloviyakh [Models and algorithms for decision making in fuzzy conditions]. Tyumen: Tyumenskii gos. Universitet Publ., 2000, 352 p. (rus)

6. Sanitarno-zashchitnyye zony $i$ sanitarnaya klassifikatsiya predpriyatiy, sooruzheniy i inykh obyektov [Sanitary protection zones and sanitary classification of enterprises, buildings and other facilities]. SanPiN 2.2.1/2.1.1.1200-03, zareg. v Ministerstve yustitsii RF 29.04.2003, No. 4459. (rus)

7. Khristodulo O.I., Salimzyanov I.F., Gareyeva N.R. Mnogokriterialnaya matematicheskaya model pozitsionirovaniya obyektov tekhnogennoy opasnosti na osnove nechetkoy logiki [Multicriteria mathematical model of positioning objects man-made hazards on the basis of fuzzy logic]. Geoinformatsionnyye tekhnologii $v$ proyektirovanii $i$ sozdanii korporativnykh informatsionnykh system [Geoinformation technologies in the design and construction of corporate information systems], Ufa: UGATU Publ., 2015, Pp. 55-64. (rus)

8. Zade L. Ponyatiye lingvisticheskoy peremennoy $i$ yego primeneniye $k$ prinyatiyu priblizhennykh resheniy [The concept of linguistic variable and its application to decision-making close], Moscow: Mir Publ., 1976, 166 p. (rus)

9. Shtovba S.D. Vvedeniye v teoriyu nechetkikh mnozhestv $i$ nechetkuyu logiku [Introduction to the theory of fuzzy sets and fuzzy logic], Vinnitsa: Kontinent-Prim Publ, 2003, 198 p. (rus)

10. Zak Yu.A. Prinyatiye resheniy $v$ usloviyakh nechetkikh $i$ razmytykh dannykh: Fuzzy-tekhnologii [Decision making under fuzzy and fuzzy data: Fuzzytechnology], Moscow: Knizhnyy dom «LIBROKOM» Publ., 2013, 352 p. (rus)

11. Pavlov S.V., Davletbakova Z.L., Abdullin A.Kh. Informatsionnaya sistema podderzhki prinyatiya resheniy po upravleniyu otkhodami na territorii Respubliki Bashkortostan na osnove bazy 
nechetkikh znaniy [Information Decision Support System for Waste Management in the Republic of Bashkortostan on the basis of fuzzy knowledge base].
Elektrotekhnicheskiye $i$ informatsionnyye kompleksy $i$ sistemy [Electrical and information systems and systems], 2013, No. 4, Vol. 9, Pp. 113-120. (rus)

ХРИСТОДУЛО Ольга Игоревна - заведующий кафедрой геоинформационных систем Уфимского государственного авиационного технического университета, доктор технических наук.

450000, Россия, Республика Башкортостан, г. Уфа, ул. К. Маркса, д. 12.

E-mail: o-hristodulo@mail.ru

KHRISTODULO Olga I. Ufa State Aviation Technical University.

450000, Karl Marx Str. 12, Ufa, Republic of Bashkortostan, Russia.

E-mail: o-hristodulo@mail.ru

САЛИМЗЯНОВ Ильяс Фаритович - аспирант кафедры геоинформаиионных систем Уфимского государственного авиационного технического университета.

450000, Россия, Республика Башкортостан, г. Уфа, ул. К. Маркса, д. 12.

E-mail: mauler@yandex.ru

SALIMZYNOV Iliyas F. Ufa State Aviation Technical University.

450000, Karl Marx Str. 12, Ufa, Republic of Bashkortostan, Russia.

E-mail: mau1er@yandex.ru

ГАРЕЕВА Наиля Рифовна - студентка кафедры геоинформационных систем Уфимского государственного авиащионного технического университета.

450000, Россия, Республика Башкортостан, г. Уфа, ул. К. Маркса, д. 12.

E-mail: nailyarg@yandex.ru

GAREEVA Nailya R. Ufa State Aviation Technical University.

450000, Karl Marx Str. 12, Ufa, Republic of Bashkortostan, Russia.

E-mail: nailyarg@yandex.ru 